\title{
Guía para el uso del Localizador de foramen
}

\section{Apex Locator: A Reliable and Easy Guide}

Marco Antonio Meza Pacheco DDS1

1. Profesor Adjunto, Sede de Occidente, Universidad de Costa Rica, Costa Rica

Autor para correspondencia: Dr. Marco Antonio Meza Pacheco - fameco5@yahoo.es

Recibido: 18-III-2014

Aceptado: 10-XI-2015

Publicado Online First: 18-XI-2015

D0I: http://dx.doi.org/10.15517/ijds.v0i0.22043

\section{RESUMEN}

En la actualidad es cada vez más la aceptación y confiabilidad que tienen los localizadores apicales en las consultas endodónticas, ya que cada vez logran mediciones más exactas de la longitud de trabajo al localizar no solamente la constricción apical sino que también determina la salida del foramen apical y, por ende se puede predecir más certeramente el resultado de la terapia endodóntica; todo esto gracias a un largo período de desarrollo y perfeccionamiento de estos aparatos desde mediados del siglo pasado, así como también suficientes estudios que avalan resultados positivos y confiables. La endodoncia moderna no puede ni debe dejar de lado el uso los localizadores de ápice ya que como hemos mencionado es una herramienta fundamental no solo en la determinación de la longitud de trabajo sino también en el diagnóstico de perforaciones y fracturas. Sin embargo, así como tiene sus ventajas también tiene sus desventajas, las cuales hay que tener presentes al momento de utilizar estos aparatos, sin olvidar tampoco seguir al pie de la letra las indicaciones del fabricante y mantenerse actualizado respecto a los usos, indicaciones y contradicciones que arrojen estudios recientes.

\section{PALABRAS CLAVE}

Localizador de apice, Longitud de trabajo, Constricción apical.

\section{ABSTRACT}

Now days is more the trust we can have to the Apex-locator in endodontics. Now we can expect more accuracy results working length measure, not only of the apical constriction but the total length of the roots. Endodontics can't left behind the use of the Apex-locator, because of its useful work, not only on the determination of the works length, also in the diagnostic of perforations or fractures. Even do, this article displays a reliable and simple guide in the use of apex locator during enodontic treatment.

\section{KEYWORDS}

Apex-locator, Working length, Apical constriction.

MEZA M., 2015: Guía para el uso del Localizador de foramen.-ODOVTOS-Int. J. Dental Sc., 17-1 (January-April): 31-40. 


\section{INTRODUCCIÓN}

Desde principios del siglo pasado, cuando Custer inicio el uso de aparatos eléctricos para la medición de los conductos radiculares hasta nuestra era, los localizadores de ápice han venido tomando fuerza al aumentar la confiabilidad de sus mediciones con cada una de sus nuevas generaciones que ha venido apareciendo vertiginosamente, al punto de ser una herramienta invaluable en los consultorios endodónticos gracias a los altos niveles de precisión con los que se cuenta hoy día, llegando a ser de hasta un $97.4 \%$ de acierto en sus mediciones aún en condiciones en las cuales antes hubiera sido impensable lograr una medición certera, como en presencia de sangre, hipoclorito incluso sangre o pus.

Considerando que uno de los puntos clave del éxito en la endodoncia recae en una correcta y exacta determinación de la longitud de trabajo 0 conductometría, sabemos que el uso de todos los instrumentos diagnósticos a nuestra disposición para lograrlo serán de gran ayuda y deben ser siempre utilizados por el clínico en pos de buscar resultados óptimos en su tratamiento y así poder brindar al paciente el mejor pronóstico posible. Teniendo clara la importancia de trabajar a una correcta longitud de trabajo, la conductometría se define como la técnica empleada para encontrar esta longitud de trabajo, que luego será utilizada en la instrumentación del conducto radicular y, que debe ajustarse al nivel de la unión del conducto cementario con el conducto dentinario o área CDC. (1) límite apical de tratamiento de conductos.

Las radiografías son importante porque nos dan información de la morfología de la pieza a tratar y las estructuras vecinas, sin embargo presentar varios inconvenientes:

- El foramen con frecuencia no coincide con el vértice radiográfico y si está lateral no siempre es revelado con la radiografía.
- Complejidades anatómicas como dislaceraciones pueden pasar desapercibidas sobre todo las que se encuentran en dirección VL o VP.

- La superposición de estructuras puede dificultar 0 impedir la visión de la región apical.

- La subjetividad en la interpretación de la imagen radiográfica.

- Estas dificultades favorecieron el desarrollo de aparatos electrónicos con el fin de localizar el foramen apical y así determinar la longitud del conducto radicular (1).

\section{ANTECEDENTES}

La primera referencia del uso de un aparato electrónico para la medición del conducto radicular es de Custer en 1916. (2). Sunada en 1958 creó el método electrónico para determinar la longitud real del diente por medio de la diferencia de potencial electrónico entre el complejo dentinocementario y el ligamento periodontal, sin embargo los resultados no eran confiables con el conducto húmedo ya que cerraba la corriente antes de la localización del foramen, algunos de estos aparatos son el Sono-Explorer, EAC, Forameter, Neosono -D, Apex Finder, entre otros, conocidos como "de tipo resistencia" o de primera generación. Con este concepto se fabricaron varios aparatos, que tenían una medida de acierto que van del 15\% hasta el $93 \%$ aproximadamente $(1,2,3,4)$.

Ushiyama en 1983 y Yamahoka et al. en 1989 desarrollaron la segunda generación de localizadores de ápice basado en la impedancia, o sea, la capacidad de los materiales de impedir el paso de la corriente eléctrica, entre ellos se encuentra el Electronic Ohmeter y el Endocater, representaron un gran avance y reportaron altos porcentajes de acierto $(2,4)$.

En 1990 Saito \& Yamashita originaron un nuevo principio dando lugar a los localizadores de ápice de tercera generación que utilizaban tanto la resistencia como la impedancia. Utilizaron una 
corriente eléctrica alternada de dos frecuencias, de $-1 \mathrm{KHz}$ y $5 \mathrm{KHZ}$ en los primeros modelos, comprobando que las diferentes regiones del conducto tenían diferentes impedancias. Los localizadores poseen dos electrodos, uno se adapta al labio inferior del paciente y el otro se ajusta al instrumento endodóntico; con la introducción de la lima en sentido apical aumentará la discrepancia entre los valores de impedancia, siendo máxima en apical, junto con un registro en un visor y una alarma sonora que indica esta posición. Así, demostraron que en la porción coronal la impedancia era mínima y que en la constricción apical era máxima, cambiando súbitamente al llegar al tejido periapical, además para que se cumpliera esto NO era necesario que el conducto estuviera seco, siendo ésta una de las grandes ventajas de los aparatos que utilizan este principio, pudiendo ser utilizados en presencia de pus, secreciones y tejido pulpar, aumentando la confiabilidad $(2,3,4)$, incluso se afirma que "los localizadores de nueva generación se pueden utilizar en medio húmedo, incluso con líquidos electroconductores..."(4).

\section{TIPOS DE LOCALIZADORES}

Típicamente los localizadores de ápice tienen 3 componentes (5):

1. Clip o pinza de labio.

2. Clip o pinza de lima.

3. Instrumento mismo, que tiene una pantalla en la que se indica el avance de la lima hacia el ápice.

La tercera y cuarta generación de localizadores apicales, dependientes de la frecuencia los primeros (resistencia e impedancia juntas) y multi-frecuencia los de cuarta generación (por ejemplo el Apex NRG, el Element Diagnostic Unit y Apex Locator y el Root ZX que trabaja con multi-frecuencia, de 0.4 y 8khz simultáneamente), tienen índices de precisión que varían del 83\% al $97,5 \%(5,8,13,14)$. Algunos de los localizadores de ápice que se encuentran en el mercado y utilizan este principio son $(1,4)$ :

- APIT (OSADA- Japón)

- ENDEX (versión norteamericana de APIT)

- ENDEX PLUS (OSADA-E.U.A)

- JUSTY II (YOSHIDA- Japón)

- R00T ZX (J. MORITA M.F.G. Corp-Japón)

- TRI-AUTO ZX (J. MORITA M.F.G. Corp-Japón)

- DENTA PORT ZX (J. MORITA M.F.G. Corp-Japón)

- APEX FINDER AFA 7005 (ANALYTIC ENDODONTICSE.U.A)

- NEOSONO ULTIMA EZ (AMADENT -E.U.A)

- BINGO 1020 de la FORUM-Israel, distribuido en Brasil por Romibras Ltda- Río de Janeiro, RJ-Brasil.

- PROPEX (DENTSPLY/MAILLEFER-Suiza)

- DATAPEX (DENTSPLY/MAILLEFER-SUiza)

- ENDOANALIZER 8005 (ANALYTICENDODONTICSE.U.A)

- APEX LOCATOR (SYBRONENDO- sds E.U.A

- NOVAPEX (FORUM- Israel).

El Neosono Ultima EZ (End Analizer) utiliza múltiple frecuencia, tiene dos circuitos que permiten realizar mediciones en conductos húmedos y secos (1).

El Apex Finder (Anaytic Endodontics) mide los valores de impedancia en el conducto por sobre 5 señales de frecuencia; sus mejores resultados clínicos los consigue cuando se usa en combinación con hipoclorito de sodio (1).

El Endex Plus (Osada Inc.Ca) es un excelente localizador apical gracias a su capacidad de calcular simultáneamente la relación de dos impedancias en el mismo canal usando diferentes frecuencias, de 1 a $5 \mathrm{kHz}$ y es capaz de determinar la longitud del canal en presencia de electrolitos 0 tejido pulpar vital o necrótico $(1,5)$.

El Root ZX (J. Morita) desarrollado por Kobayashi y Suda es uno de los mejores aparatos disponibles en el mercado como localizador apical. 
Usa frecuencias entre 8 y $0,4 \mathrm{kHz}$. Se puede usar en conductos húmedos, en presencia de hipoclorito de sodio, sangre, agua y anestesia. En varios estudios ha demostrado tener altos grados de confiabilidad en sus lecturas $(1,5,3)$.

Recientemente la firma J. Morita introdujo el Tri Auto ZX, que consta de una pieza de mano de baja velocidad para endodoncia combinado con la tecnología del Root ZX, dando al clínico la capacidad de monitorear electrónicamente el conducto antes, durante y después de la instrumentación. Tiene 3 funciones automáticas: (1)

- Inicio y cese automático.

- Torque en reverso automático.

- Rotación en reverso apical automático.

\section{USO DE LOS LOCALIZADORES DE ÁPICE}

Los localizadores de ápice trabajan por medio de la impedancia, esta se define como la relación entre la tensión alterna aplicada a un circuito y la intensidad de la corriente producida. Se mide en ohmios (6). Es una magnitud que establece la relación entre la tensión y la intensidad de corriente. Es la oposición total sobre la corriente. La pared dentinaria del conducto radicular exhibe una baja conductividad eléctrica, de esta forma a medida que se aproxima al tercio apical, la capa de tejido dentinario se vuelve menos espesa y disminuye su capacidad de aislamiento eléctrico. Esta disminución gradual se interpreta eléctricamente como una disminución de la impedancia de la dentina. Los aparatos basados en el método de frecuencia tienen una calibración tal, que permite indicar la variación de los valores relativos a la impedancia (cociente 0 diferencia) de la región apical, y permiten colocar la punta del instrumento a aproximadamente $1 \mathrm{~mm}$ del foramen apical, posición idónea para la terminación apical de la endodoncia. La impedancia es la capacidad que los materiales exhiben de resistir al paso de la corriente eléctrica (7).

En el conducto radicular se conecta el instrumento (lima) mediante una pinza al aparato de medición. A través de la lima se dirige una corriente hacia el conducto radicular. Como electrodo contrario se usa un gancho en el labio del paciente. Los aparatos indican el orificio apical cuando se alcanza un valor de resistencia (a la corriente) prefijado, no obstante ni los aparatos más modernos pueden determinar el área CDC, solamente localizan el cambio de impedancia cuando la lima pasa al tejido periodontal del ápice. La caída de la tensión entre los dos electrodos (lima y gancho del paciente) se indica en una escala de diodos luminosos, sin embargo esta indicación no corresponde a la distancia real desde la lima al orificio (8).

En dientes con un contenido del conducto no extirpado se observó que se originaba en su circuito de medición una resistencia de unos 6,5 kiloohmios (8). La presencia de pulpa inflamada en el trayecto del conducto radicular, dificulta las mediciones electrónicas. Clínicamente se observa que tales mediciones son más fáciles de evaluar en conductos con contenido pulpar necrótico 0 también en casos de re tratamiento, siempre y cuando anteriormente se retire correctamente el contenido. Por lo tanto, se recomienda realizar una pulpectomía parcial, seguida de abundante irrigación con hipoclorito de sodio, para efectuar la medición sin la interferencia de la pulpa inflamada en el conducto (7).

Se ha demostrado que la presencia de pulpas con procesos inflamatorios agudos tienden a alterar las mediciones, pues este tejido presenta su potencial de conductividad eléctrica alterado, 
exhibe valores superiores a lo calibrado en los aparatos y distorsiona su respuesta (7).

En los casos de ápice incompleto, reabsorción apical avanzada o de sobre instrumentación, la constricción apical puede estar perjudicada 0 ausente, y alterar la conformación eléctrica del conducto radicular (7).

Como media, el mejor de estos instrumentos proporciona mediciones precisas hasta $0,5 \mathrm{~mm}$ del ápice. Por lo tanto para tener una medición "de seguridad" se debe estar a $1 \mathrm{~mm}$ de la cifra proporcionada por el aparato y confirmarlo con una radiografía, sin embargo las mediciones electrónicas proporcionan estimaciones más exactas de la longitud de trabajo que la habitual sustracción de $2 \mathrm{~mm}$ en la radiografía pre operatoria (5).

Las consideraciones que debe tener el clínico, antes y durante la utilización de dispositivos electrónicos, para establecer correctamente la longitud de trabajo se enumeran a continuación $(4,58,9)$ :

- Se recomienda el uso diario para mejorar su manipulación con la práctica.

- La solución de irrigación solo debe permanecer en los conductos, sin inundar la cámara.

- El diente debe estar correctamente aislado ya que si tiene alguna comunicación con la cavidad pulpar o tejido periodontal (por caries 0 trepanaciones) los resultados no serán fiables.

- La lectura se considera confiable cuando el movimiento de la barra del monitor es estable y solo se mueve junto y acorde al movimiento de la lima. Cuando esto sucede Irrigue, aspire el exceso de irrigante y reinicie el procedimiento. Controle la presencia de contacto entre la lima y las restauraciones metálicas. Haga una prueba preliminar tocando los electrodos. Controle principalmente la presencia de exceso de tejido pulpar, en caso de pulpa irreversiblemente inflamada. Controle la carga de la batería.

- Es importante hacer las mediciones con limas manuales ya que el constante movimiento de las limas rotatorias afectan negativamente el éxito de las determinaciones en la longitud de trabajo.

- La lima no debe estar en contacto con metales. Las restauraciones de amalgama deben retirarse ya que además de interferencias con el localizador de ápice pueden crear filtración marginal además de que fragmentos de metal pueden llegar al interior del conducto.

- Para obtener la medición electrónica el aparato debe, necesariamente, llegar a la constricción apical. En casos de retratamiento se debe eliminar toda la gutapercha antes de hacer la medición. Además considerar que los conductos amplios pueden cerrar antes el circuito.

- Estos aparatos también se utilizan para la localización y diagnóstico de fracturas radiculares o perforaciones, ya que comportan como si hubieran llegado a tejido periodontal.

- Se deben seguir las instrucciones del fabricante en cada caso.

- IMPORTANTE: la medición con esto aparatos no excluye la toma de radiografías para corroborar el resultado.

- Se recomienda no dejar el aparato con las baterías por mucho tiempo. Al terminar la medición, desconecte el aparato para evitar el desperdicio de la batería.

- Guarde siempre el cable del equipamiento recto, o envolviéndolo en un carretel grande tipo línea de pescar. Doblar el cable puede romper uno de los hilos que pasan por dentro y dañar el equipamiento.

- En casos de dientes con vitalidad pulpar, realizar una pulpectomía parcial removiendo, por lo menos dos tercios del volumen del tejido pulpar. Clínicamente se nota que en los casos de pulpa irreversiblemente inflamada, la colocación del instrumento en el tercio cervical puede indicar 
una medición como si estuviera en el punto próximo a la constricción apical. Cuando se remueve parcialmente el tejido, con irrigación abundante y se aspira el exceso de líquido irrigante, la medición tiende a volver a lo normal.

- El instrumento utilizado para la medición debe ser compatible con el diámetro anatómico del conducto.

- Los electrodos (portalima y polo colgante del labio) deben estar libres de oxidaciones resultantes del contacto con solución irrigante. Este detalle evita parcialmente la oxidación.

\section{TÉCNICA DE CONDUCTOMETRÍA ELECTRÓNICA (7)}

- Después del acceso coronal y del aislamiento absoluto del campo operatorio, el conducto radicular debe irrigarse copiosamente con solución de hipoclorito de sodio, con las diversas concentraciones indicadas para caso.

- Certifíquese que los cables estén conectados correctamente.

- Con el cable conectado al aparato, prender el aparato antes de instalar los electrodos en el intermediario del instrumento y en la comisura labial del paciente.

- Antes de colocar el instrumento en el interior del conducto, se deben observar algunos detalles:

- Ejecutar un toque entre los electrodos (el de la lima y el colgante del labio). Esto cerrara el circuito eléctrico, presentando una lectura de impedancia baja. Este procedimiento deberá resultar en un movimiento del marcador de la pantalla del equipamiento, indicando un punto próximo al que se refiere a la posición del foramen apical.

- Comprobar si el diente, después del acceso, está bien aislado y si las restauraciones metálicas no están proyectadas sobre las entradas de los conductos radiculares. Las restauraciones metálicas desvían el circuito, disminuyendo la impedancia y dando un resultado falso positivo.

- La carga de la batería debe estar completa. Equipamientos de precisión como localizadores electrónicos de foramen no funcionan correctamente con carga parcial.

- Para casos de biopulpectomía: una pulpectomía parcial debe realizarse antes de la medición, para posibilitar la ejecución de la mensuración electrónica. Esta pulpectomía parcial debe tener su límite aproximadamente, $5 \mathrm{~mm}$ antes de la longitud del diente en la radiografía, establecido por la medición de la imagen en la radiografía para diagnostico. En caso de hemorragia, ésta no puede exceder el límite de la entrada o de las entradas de los conductos. En casos extremos, una torunda de algodón se puede colocar en el interior de la cámara pulpar, para evitar que el sangrado dificulte la lectura. El instrumento puede introducirse al lado de la bolita de algodón.

- Para los casos de necrosis: la solución de hipoclorito de sodio provocara una limpieza inicial de los restos necróticos del interior de la cámara pulpar. Después de la fase inicial de instrumentación progresiva, limitada apicalmente a $5 \mathrm{~mm}$ antes de ápice radiográfico medido en la radiografía para diagnostico, un instrumento compatible con el diámetro anatómico se introduce suave, sin excesiva presión apical. A este instrumento estará conectado el polo de la lima del aparato. El material irrigante debe estar ausente de la cámara pulpar, limitándose a la embocadura o embocaduras de los conductos.

- Electrodo, se coloca en la comisura labial del paciente.

- Cuando se ha optado por la técnica de instrumentación progresiva en sentido coronoápice (crown-down), los instrumentos con mas calibres pueden utilizarse hasta un límite de $5 \mathrm{~mm}$ anteriores a la medición inicial, hecha a partir de la radiografía para diagnostico. En este caso se acopla el portalima al instrumento en la secuencia operatoria y se hace la lectura. Es importante que los conductos radiculares estén con solución irrigante, y la cámara pulpar no contenga exceso de líquido irrigante.

- El instrumento endodóntico elegido para ejecutar el cateterismo y la conductometría electrónica, 
debe ser $5 \mathrm{~mm}$ mayor que la longitud de trabajo provisional. Esto debido a que es necesario un espacio libre para colocar el gancho en el intermediario y el tope de goma.

- Introducir el instrumento en el interior del conducto radicular. Certifíquese que el instrumento se ajusta a las paredes internas. Instrumentos muy finos pueden dar resultados falsos positivos. Utilizar instrumentos de diámetro próximo al diámetro anatómico.

- Introducir apicalmente la lima, haciéndola girar suavemente en el sentido horario, percibiendo el inicio del movimiento en el indicador del visor. Este movimiento tendrá la exacta velocidad de la penetración del instrumento en el interior del conducto. En dirección al foramen apical.

- Al aproximarse a las marcas finales, una alarma sonora se acciona. Continuar con el instrumento en sentido apical hasta que la alarma toque de forma continua, posicionando la marca que corresponde a la posición de la salida del foramen. Al llegar a esta marca retroceder el instrumento hasta el punto que corresponde a la posición de la constricción apical. El sonido de la alarma volverá a ser intermitente.

- En este momento, el operador debe proceder a marcar la Longitud Real de Trabajo deslizando el tope hasta el punto de referencia elegido.

VENTAJAS $(7,9,10)$.

- La localización electrónica del ápice es más fácil y rápida que con medios radiográficos.

- La longitud de trabajo es fácilmente reproducible son necesidad de exponer al paciente a radiación.

- El localizador se puede usar para corroborar la longitud de trabajo en cualquier momento del procedimiento.

- La determinación de la longitud de trabajo es más certera y predecible.

- Los localizadores modernos pueden localizar no solo el foramen apical sino también la última constricción apical en sentido apical, lugar idóneo de terminación de la endodoncia en este sentido.
- Los localizadores de ápice pueden diagnosticar perforaciones.

- Para el diagnóstico de fracturas radiculares son una herramienta muy útil.

- Usada junto con la verificación radiográfica, la determinación exitosa de la longitud de trabajo aumenta al máximo.

DESVENTAJAS $(4,7,9)$.

- Se debe tener práctica para optimizar su uso.

- En algunos casos las lecturas pueden ser incorrectas, la disfunción del aparato puede ser reconocida cuando la escala de barras en la pantalla del localizador está inestable y brinca de una medición a otra inestablemente o cuando no aparece completa.

- NO SE DEBE UTILIZAR ESTE APARATO EN PACIENTES PORTADORES DE MARCAPASOS.

- La obliteración parcial o total de los conductos puede interferir con la correcta lectura del aparto.

- En retratamientos se debe eliminar bien el contenido del conducto antes de usar el localizador de ápice ya que puede comportarse como si estuviera parcial o totalmente obliterado dando mediciones incorrectas, además considerar que los retratamientos tiene mayor probabilidad de presentar obliteración de los conductos.

- En casos de pulpitis agudas irreversibles puede dar falsas mediciones.

\section{DISCUSIÓN}

El localizador de ápice Root ZX presenta un alto nivel de confiabilidad en sus lecturas respecto a la longitud de trabajo; el acierto in vivo fue de un $88.9 \%$, sin sufrir interferencias en presencia de hipoclorito de sodio al 1\% (1). Sin embargo, se debe tener presente que los conductos amplios en el tercio apical tienden a no proporcionar medidas tan exactas. (11). Además destacar la importancia del efecto negativo que tiene la obliteración de los conductos en la medición electrónica de ellos, ya que en un estudio (9) las mediciones fueron 
inconsistentes en presencia de una obliteración parcial o total de los conductos, probablemente por la interrupción del circuito eléctrico. Además, las mediciones no se vieron afectadas por la presencia de restauraciones metálicas, siempre que el diente estuviera bien aislado con dique de hule y eliminado el exceso de irrigante de la cámara pulpar. Tampoco se demostró influencia negativa en las mediciones cuando la pulpa se encontraba vital 0 necrótica. Para hacer estas mediciones usaron dos localizadores de ápice: el Root ZX y el Raypex5 (VDW, Munich, Germany); en conjunto los dos tipos de localizador tuvieron un grado de acierto del $85 \%$, pero al excluir a las piezas que presentaban obliteración el grado de acierto aumento a un 96\%. El éxito en las medidas del Root ZX fue significativamente mayor que el del Raypex5, sin embargo esta diferencia fue significativa estadísticamente y no clínicamente.

En otro estudio (12) se compararon tres localizadores de ápice distintos a saber el Root ZX (J. Morita), el Elements Apex Locator (ELE) (SybronEndo) y el Precision Apex Locator (PAL) (Brasseler, USA). El localizador de ápice ELE es de cuarta generación con una exactitud de medición reportada de un $94.28 \%$ según Plotio et al. El PAL es un nuevo localizador de ápice que no ha sido estudiado al día de hoy y, por último el Root ZX es un localizador de ápice de tercera generación con una tasa de éxito reportada del 82\%-100\% y, Shabahang et al. reporta un éxito de un $96.2 \%$ para este localizador. Actualmente está en el mercado la versión mejorada del Root ZX, el Root ZX II. Guise et al. determinaron que sí hubo diferencias significativas en el porcentaje de éxito de las mediciones entre el ELE y el PAL comparándolos con el Root ZX, sin embargo no hubo diferencias entre el PAL y el ELE. Se determinó la longitud de trabajo tal y como los sugieren los fabricantes: utilizando el localizador para determinar foramen apical y restando $0.5 \mathrm{~mm}$ y de esta forma el Root ZX obtuvo un acierto del $97.5 \%$ y en los casos de error se mostró corto en relación a la medición mientras que el ELE y el PAL tuvieron un éxito del $90 \%$ y $95 \%$ respectivamente y al fallar lo hicieron mostrando mediciones sobre-extendidas (12). Estos resultados respecto al éxito del Root ZX (12) se confirman con otra investiagción. (10) en un estudio en el que compararon cuatro tipos diferentes de localizadores: el Root ZX, Elements Diagnostic Unit y Apex Locator, el Mini Apex Locator y el Apex DSP. Concluyeron que el Root ZX junto con el Mini Apex Locator aumentaron significativamente la precisión en la medición de las longitudes de trabajo, aunque todos los localizadores tuvieron un grado aceptable de acierto con rangos de +/0.5mm, a excepción del Apex DSP (10).

Asimismo se ha analizado el desempeño del localizador de ápice ProPex se encontró que daba una lectura certera en un $80 \%$ de los casos a $0.5 \mathrm{~mm}$ de la longitud de trabajo y, en un $95 \%$ a $1 \mathrm{~mm}$ de la longitud de trabajo (13). Además recomendaron su uso con los conductos libres de humedad ya que en presencia de ella las lecturas no son tan precisas.

Por último recientemente compararon tres localizadores de ápice de cuarta generación, usando el Root ZX II, el Apex NRG y el Mini Apex Locator con limas rotatorias de Ni-Ti. El acierto en la determinación del menor diámetro en el conducto, usando instrumental rotatorio de $\mathrm{Ni}-\mathrm{Ti}$, fue de $50 \%$, 46.43\% y $39.9 \%$ respectivamente, sin embargo el éxito en las mediciones aumentaba significativamente cuando la medición se realizaba con limas manuales (14). 


\section{CONCLUSIONES}

Al realizar el tratamiento de conductos, la conductometría juega un papel muy importante para determinar el éxito del tratamiento endodóntico.

Tradicionalmente se ha determinado la longitud de trabajo por medios radiográficos sin embargo presenta ciertos inconvenientes, como por ejemplo la superposición de estructuras 0 el hecho de que generalmente el ápice radiográfico no coincide con el ápice anatómico.

La primera referencia del uso de un aparato electrónico para la medición del conducto radicular es de Custer en 1916, luego en 1958 Sunada creó los localizadores de primera generación. Posteriormente en 1989 Ushiyama y Yamahoka et al. desarrollaron la segunda generación de localizadores, y por último en 1990 Saito \& Yamashita originaron un nuevo principio dando lugar a los localizadores de ápice de tercera generación que utilizaban tanto la resistencia como la impedancia.

En la actualidad los localizadores de ápice son más precisos que los usados a mediados del siglo pasado, actualmente logran mediciones precisas incluso en presencia de humedad, sangre o pus.

Los localizadores de ápice además de ayudarnos a determinar la longitud de trabajo con mayores índices de éxito, también son una gran herramienta para el diagnóstico de otras condiciones como fracturas radiculares o perforaciones.

Los localizadores de ápice indican no solo la posición de la constricción apical sino que también indican la posición del foramen apical; mientras que el análisis radiográfico interpreta solamente la posición del ápice radiográfico.

Los localizadores de primera generación usaron la resistencia como medio para el diagnóstico, luego los de segunda generación usaron la impedancia de los tejidos. A partir de estos dos conceptos surgieron los localizadores de ápice de tercera generación que utilizan la frecuencia (resistencia y impedancia juntas), y luego los de cuarta generación que utilizan la multi-frecuencia para realizar el diagnóstico.

Aún queda por perfeccionar los localizadores apicales, sobre todo en presencia de pulpitis agudas, ya que se ha demostrado que la lectura es afectada en éste estado de la pulpa.

Para lograr lecturas acertadas de los localizadores de ápice es de vital importancia que el clínico considere cuidadosamente todas las indicaciones que indica el fabricante.

RECORDAR LA CONTRAIDICACIÓN DEL USO DE ESTOS APARATOS EN PACIENTES CON MARCAPASOS.

En la mayoría de los artículos revisados se concluyó que el localizador de ápice que arroja mejores resultados es el Root ZX de la casa J. Morita.

Los localizadores electrónicos de foramen ya ocupan un lugar destacado en el contexto de la investigación y de la clínica endodóntica.

\section{BIBLIOGRAFÍA}

1. Villena M, H. Terapia Pulpar. Lima, Perú: Universidad Peruana Cayetano Heredia; 2001.

2. Soares, IJ. Goldberg, F. Endodoncia. Técnicas y fundamentos. Buenos Aires, Argentina: Editorial Panamericana; 2003.

3. Baldi JV, Victorino FR, Bernardes RA et al, Influence of Embedding Media on the Assessment of Electronic Apex Locator. JOE. 2007; Vol 33; N4: 476-479.

4. Leonardo, MR. 2005. Endodoncia: Tratamiento de Conductos Radiculares. Principios Técnicos 
y Biológicos. Volumen 1. Brasil: Editorial Artes Médicas; 2005.

5. Cohen, S. Burns, R. C. Vías de la Pulpa. 8va edi. España; Edit. Mosby; 2010.

6. Diccionario de la Real Academia Española. (En línea). España: Real Academia Española, 2014. (Fecha de acceso 14 de agosto de 2015). URL disponible en http://www.rae.es.

7. Leonardo, M.R. 2005. Endodoncia: Tratamiento de Conductos Radiculares. Principios Técnicos y Biológicos. Volumen 2. Brasil: Editorial Artes Médicas: 2005.

8. Beer, R. Baumann, M.A. Kim, S. Atlas de Endodoncia. Barcelona: MASSON; 2000.

9. ElAyouti A., Eleftheria D., Ohmer J. et al, Consistency of Apex Locaro Function: AClinical Study. JOE. 2009, Vol. 35; No2:179-181.

10. Janolio de Camargo E., Zapata R.O., Medeiros PL et al. Influence of Preflaring on the Accuracy of Length Determination With Four Electronic Apex Locators. JOE. 2009. Vol. 35, No9: 1300-1302.

11. Herrera M., Ábalos C., Planas A. J., Llamas R., .Influence of Apical Constriction Diameter on Root ZX Apex Locator Precision. JOE. 2007; Vol. 33, No8: 995-997.

12. Guise G.M., Goodell G.G., Imamura GM, In Vitro Comparision of Three Electronic Apex Locator. JOE. 2010. Vol. 36, No2: 279-281.

13. Özsezer E., Ínan U., Aydn U. In Vivo Evaluation of ProPex Electronic Apex Locator. JOE. 2007; Vol.33, No8: 974-977.

14. Siu C., Marshall G., Baumgartner C. An In Vivo Comparision of the Root ZX II, the Apex NRG XFR, and Mini Apex Locator by Using Rotary Nickel-Titanium Files. JOE. 2009; Vol. 35, № 7: 962-965. 